\title{
High-resolution quantitative trait locus mapping for body weight in mice by recombinant progeny testing
}

\author{
XIAOJUN LIU ${ }^{1}$, FIONA OLIVER ${ }^{1}$, STEVE D. M. BROWN ${ }^{2}$, \\ PAUL DENNY ${ }^{2}$ AND PETER D. KEIGHTLEY ${ }^{1 *}$ \\ ${ }^{1}$ Institute of Cell, Animal and Population Biology, University of Edinburgh, West Mains Road, Edinburgh EH9 3JT, UK \\ ${ }^{2}$ MRC UK Mouse Genome Centre and Mammalian Genetics Unit, Harwell, Oxfordshire OX11 ORD, UK \\ (Received 30 June 2000 and in revised form 30 October 2000)
}

\begin{abstract}
Summary
A major obstacle to the positional cloning of quantitative trait loci (QTLs) lies in resolving genetic factors whose allelic effects are blurred by environmental and background genetic variation. We investigate a fine-mapping approach that combines the use of an interval-specific congenic strain with progeny testing of recombinants for markers flanking a QTL. We apply the approach to map a murine QTL with an approximately $20 \%$ effect on growth rate by progeny testing 39 recombinants in a $12 \mathrm{cM}$ region of the $\mathrm{X}$ chromosome. We use a likelihood analysis in an attempt to maximize the information on QTL map location and effect. The major X-linked effect is mapped to an approximately $2 \mathrm{cM}$ region flanked by markers about $5 \mathrm{cM}$ apart, outside which LOD support for the QTL drops extremely steeply by about 80 . Nearly unambiguous assignment of the QTL genotypic state is obtained for each recombinant. The resolution of individual recombinants in the region is therefore sufficiently high to facilitate the positional cloning of the locus, although progress has been hampered because the genomic region containing the QTL shows an exceptionally low level of polymorphism in comparison with recent studies.
\end{abstract}

\section{Introduction}

The experimental dissection of quantitative traits is a multi-stage process, typically involving coarse genome-wide linkage mapping, fine-mapping of specific regions, and with the aim of positionally cloning and characterizing quantitative trait loci (QTLs). The widespread availability of molecular genetic markers has led to a proliferation of QTL mapping projects in diverse species. In many of these, attention is now directed at fine-mapping in those regions of the genome that have shown significant associations in genome-wide scans. Although in most cases the ultimate goal of positionally cloning QTLs has remained elusive, the goal has recently been achieved for a QTL $f w 2.2$ influencing fruit weight in tomato (Frary et al., 2000).

Several strategies have been suggested to increase the resolution of QTL detection for high-resolution mapping (reviewed by Darvasi, 1998). Here, we use

\footnotetext{
* Corresponding author. Tel: +44 (0)131 650 5443. Fax: +44 (0)1316506564. e-mail; p.keightley@ed.ac.uk
}

progeny testing of recombinants for fine-mapping in combination with an interval-specific congenic strain (ISCS). The basis of progeny testing is to find recombinants in an interval believed to contain a QTL, to propagate these recombinants, then to measure the phenotypes of their progeny in order to estimate precisely the genotypic value of each recombinant. If a QTL co-segregates with a marker, its presence is detected on the basis of a difference between marker class means. Breese \& Mather (1957) first used ISCs to map QTLs affecting bristle score in Drosophila, while Thoday (1961) tested for the presence of distinct segregating factors for bristle number in Drosophila between pairs of visible markers that had been allowed to recombine. Shrimpton \& Robertson (1988) were the first to combine the use of ISCSs with progeny testing of recombinants, again to map bristle number factors in Drosophila.

Our experiment focuses on an X-linked QTL affecting body weight of mice in selection lines derived from a cross between two inbreds and an outbred (Sharp et al., 1984). The presence of a large X-linked 
effect was first suggested in reciprocal crosses between lines selected high and low for body size, in which an unexpectedly high fraction $(\sim 25 \%)$ of the selection response was associated with the $\mathrm{X}$ chromosome (Hastings \& Veerkamp, 1993). QTL mapping experiments in an F2 population from the high $\times$ low line (Rance et al., 1997a), and in series of backcross lines (Rance et al., 1997b), subsequently confirmed that there was a large additive contribution from the $\mathrm{X}$ chromosome, and suggested that the effect was caused by a single locus that mapped between the microsatellite markers DXMit50 and DXMit25, which are approximately $12 \mathrm{cM}$ apart at the proximal end of the chromosome. Backcrossing to produce an ISCS was continued to generation 6 using an inbred line derived from the low selection line as the recurrent parental strain, while selecting DXMit50 and DXMit25 high line alleles, and excluding high line flanking marker alleles at DXMit55 and DXMit62. The contribution from the high line autosomal genome was therefore expected to about $1.5 \%$.

For fine-mapping, we follow an approach similar to that of Shrimpton \& Robertson (1988), although we propagate recombinants by ordinary crossing, and cannot completely eliminate further recombination in the region. Additional markers between our pair of flanking markers are employed to further enhance the precision of QTL mapping. In order to reduce background variation, our starting material is an ISCS containing a short chromosome segment on an otherwise inbred background. We use likelihood to combine information on phenotypes and marker genotypes in the initial recombinant families and the progeny test litters. To test for additional genetic factors in the chromosome segment, we compare the likelihood of models with a single QTL or two QTLs, and perform a two-dimensional search of the parameter space allowing the map location and effect of each QTL to vary (Haley \& Knott, 1992). More general versions of this approach are favoured in testing for multiple QTLs (Kao et al., 1999; Weber et al., 1999).

\section{Materials and methods}

\section{(i) Microsatellite genotyping}

Genomic DNA was extracted from either tail clip or spleen tissue by standard methods. Microsatellite genotyping was carried out based on the protocol described by Routman \& Cheverud (1994). In cases where the difference in PCR product sizes was greater than about $6 \mathrm{bp}$, products were separated on $20 \mathrm{~cm}$ long, vertical running $6 \%$ polyacrylamide gels for $2-3 \mathrm{~h}$ at $200 \mathrm{~V}$. Gels were stained by ethidium bromide and photographed under ultraviolet light for scoring. Microsatellites that were not informative on $20 \mathrm{~cm}$ gels were tested on higher-resolution sequencing-type gels. PCR products, in which one PCR primer was labelled with ${ }^{32} \mathrm{P}$, were separated on $6 \%$ denaturing polyacrylamide gels for $2-3 \mathrm{~h}$ at $80 \mathrm{~W}$. Gels were transferred to a sheet of Whatman 3MM paper, dried, then autoradiographed for $16-24 \mathrm{~h}$ at $-70^{\circ} \mathrm{C}$.

\section{(ii) DNA sequencing}

Several regions of DNA that map close to the QTL were sequenced in an effort to find new polymorphic markers. Introns 4-8 of the Hprt gene were cloned into a pCR2.1-TOPO vector using the TOPO TA Cloning kit (Invitrogen Corporation). The PCR products of sequence-tagged sites (STS: short segments of DNA specifically amplified by PCR) and microsatellites, or DNA extracted from the clones, were sequenced according to standard protocols using the ABI Big Dye Terminator Cycle Sequencing Ready Reaction Kit (Perkin-Elmer, Applied Biosystems) on an ABI 377 sequencer. Both strands of DNA were sequenced, and any putative differences were resequenced.

\section{(iii) Recombinant progeny testing}

To fine-map the QTL, heterozygous ISCS females (HL) were backcrossed to inbred low line males (L), in order to generate individuals recombinant between DXMit50 and DXMit25 for progeny testing. The progeny test involved crossing males $\left(\mathrm{H}^{\prime}\right)$ or females $\left(\mathrm{H}^{\prime} \mathrm{L}\right)$ carrying a recombinant segment to individuals from the inbred low line in order to generate families segregating for the segment among hemizygous male $\left(\mathrm{H}^{\prime}\right.$ or $\left.\mathrm{L}\right)$ and heterozygous female $\left(\mathrm{H}^{\prime} \mathrm{L}\right.$ or $\left.\mathrm{LL}\right)$ progeny. Body weights at 6 weeks of age of the progeny from these segregating families were recorded along with the genotypes for the flanking markers. The locations of recombination break-points for each recombinant type were then established using further markers. In the first phase of the experiment 33 recombinants were found in 268 individuals typed. It quickly became clear that the QTL mapped to the interval flanked by DXMit226 and DXMit68, which maps between DXMit50 and DXMit25, so progeny testing efforts were concentrated on the recombinants between the inner two markers, with the aim of breeding at least 6 families per recombinant. Six further such recombinants between DXMit226 and DXMit68 in 125 individuals were also included in the progeny test. The map locations of the above-named markers and various others that were subsequently used are illustrated in Fig. 1.

\section{(iv) Interval mapping by maximum likelihood}

For a single-QTL model, an interval mapping algorithm similar to one previously described (Keightley et al., 1998), and based on Knott \& Haley (1992), was 


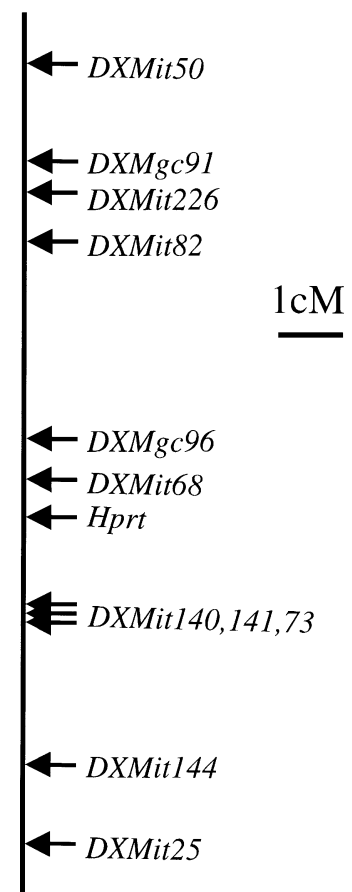

Fig. 1. Genetic map of chromosome X segment showing the approximate positions of various markers mentioned in the text.

used to estimate the effect and map location of a QTL using the progeny test data. In the progeny test scheme, each recombinant type is replicated across several litters, and, for simplicity, further recombination events after the initial recombination are ignored. The effect of ignoring these recombinants is to add noise, and therefore to reduce precision, but this becomes of decreasing importance as the length of the target interval for progeny testing is reduced through the use of additional markers. Taking male progeny as an example, a QTL with allelic effects $a_{1}$, (genotype $\mathrm{H}$ ) or $a_{2}=0$ (genotype $\mathrm{L}$ ) was assumed to be at position $Q$ in an interval flanked by a pair of markers. Uniform recombination between pairs of markers was assumed. An equation for the likelihood of recombinant type $i$ comprising $N_{i}$ litters, each containing $n$, individuals, is

$$
\begin{aligned}
L_{i}= & \sum_{q=1}^{2} p\left(a_{q} \mid Q, \text { markers }\right) \\
& \times\left[\prod_{j=1}^{N_{i}} \int \frac{1}{\left(2 \pi \sigma_{b}^{2}\right)^{\frac{1}{2}}} \exp \left[-\frac{b_{j}^{2}}{2 \sigma_{b}^{2}}\right] \prod_{k=1}^{n_{j}} \frac{1}{\left(2 \pi \sigma_{w}^{2}\right)^{\frac{1}{2}}}\right. \\
& \left.\times \exp \left[-\frac{\left(y_{j k}-\mu-a_{q}-b_{j}-\mathbf{x}_{j k}^{\prime} \mathbf{f}\right)^{2}}{2 \sigma_{w}^{2}}\right] d b_{j}\right],
\end{aligned}
$$

where $p\left(a_{q} \mid Q\right.$, markers $)$ is the probability that the QTL has a genotypic effect $a_{q}$ given $Q$ and markers, the allelic state of the flanking markers; $b_{j}$ is a random litter effect, assumed to be normally distributed with mean zero and variance $\sigma_{b}^{2} ; y_{j k}$ is the phenotypic value of the $k$ th individual from litter $j, \mu$ is the population mean, $\mathbf{x}_{j k}^{\prime}$ is a design matrix for fixed effects and a covariate (see below), $\mathbf{f}$ is a vector of fixed effect and covariate values, and $\sigma_{w}^{2}$ is the residual variance, assumed to be normally distributed.

Data from males and females were analysed together, so there was a separate $a_{q}$ fitted for each sex, and a fixed effect for sex. Additionally, there was a fixed effect with two classes for parity (first litter/not first litter), and a linear covariate term for litter size. The recombinant types are independent, so the overall likelihood for the whole data set is

$$
L=\prod_{i=1}^{\text {No.types }} L_{i}
$$

The overall likelihood was maximized as a function of the parameters of the model (the two $a_{q} \mathrm{~s}, \sigma_{b}^{2}, \mu, \sigma_{w}^{2}$, the two fixed effects and the covariate) using the Simplex algorithm (Nelder \& Mead, 1965). Integration over the distribution of litter effects was carried out numerically with Gaussian quadratures. The likelihood ratio is computed by dividing $L$ for the model with the $a_{q}$ s fitted by $L$ for the model with the $a_{q}$ s set to zero, and is converted to a LOD score. This was evaluated at $0 \cdot 1 \mathrm{cM}$ intervals along the chromosome in the interval between DXMit50 and DXMit25. The maximum likelihood (ML) estimate of QTL location is the position at which the LOD score is highest; the ML estimates of QTL effects in males and females are the estimates of the $a_{q} \mathrm{~s}$ at this point. The ML procedure was tested with simulated data; mean ML estimates of QTL position and effect were close to those simulated.

The likelihood of the single-QTL model was also compared with a two-QTL model. There is little information to enable testing for the presence of more than one QTL within an interval, so each QTL was therefore assumed to be in a different interval; this assumption also greatly simplified the implementation of the model. The interval containing the recombination break-point is known for each type of recombinant, so only a small modification to likelihood equation (1) was necessary:

$$
\begin{aligned}
L_{i}= & \sum_{q 1=1}^{2} p\left(a_{q 1} \mid Q_{1}, \text { markers }\right) \\
& \times \sum_{q 2=1}^{2} p\left(a_{q 2} \mid Q_{2}, \text { markers }\right) \\
& \times\left[\prod _ { j = 1 } ^ { N _ { i } } \int \frac { 1 } { ( 2 \pi \sigma _ { b } ^ { 2 } ) ^ { \frac { 1 } { 2 } } } \operatorname { e x p } \left[-\frac{b_{j}^{2}}{2 \sigma_{b}^{2}} \prod_{k=1}^{n_{j}} \frac{1}{\left(2 \pi \sigma_{w}^{2}\right)^{\frac{1}{2}}}\right.\right. \\
& \left.\times \exp \left[-\frac{\left(y_{j k}-\mu-a_{q 1}-a_{q 2}-b_{j}-\mathbf{x}_{j k}^{\prime} \mathbf{f}\right)^{2}}{2 \sigma_{w}^{2}}\right] d b_{j}\right]
\end{aligned}
$$

where, for example, $p\left(a_{q 1} \mid Q_{1}\right.$, markers $)$ is the probability that the first QTL has a genotypic effect $a_{q 1}$ 
given $Q_{1}$, the position of the first QTL. The overall likelihood for the two-QTL model was evaluated and maximized as described for the one-QTL model, using a grid of $0.25 \mathrm{cM}$ between evaluations for both $Q_{1}$ and $Q_{2}$.

\section{Results}

(i) QTL location and effects

In an initial screen, 6 informative markers were found between DXMit50 and DXMit25, out of a total of 45 microsatellite markers typed. The map distance of these markers, calculated from the proportion of recombinants observed in the initial generation of the progeny test, are shown in Table 1.

Marker and body weight data from the initial recombinant and progeny test families were analysed by the single-QTL ML procedure described in Section 2. The LOD score plot along the region of the chromosome tested is compared with the plot obtained from analysis of our previous F2 mapping experiment (Rance et al., 1997a) in Fig. 2. The map positions of the peak LOD scores coincide closely in the two experiments, suggesting that the QTL was successfully transferred into the ISCS. The LOD score plot for the progeny test is step-like, in contrast to the plot for the F2, which is fairly smooth. This is a consequence of the increased information on each recombinant that arises from the progeny test, and allows intervals other than the one bounded by DXMit226 and DXMit68 to be excluded as candidates for containing the QTL. We concentrated effort on recombinants in the DXMit226 - DXMit68 interval as the experiment progressed (Table 1). The drops in LOD score on either side of these markers differ partly as a consequence of different numbers of recombinants containing either the proximal or distal marker.

The progeny test results are shown in greater detail along with the results of a ML analysis that only used the litters containing the initial recombinants in Fig. 3. Progeny testing led to large increases in the heights of the steps in the LOD score profile. Although the data from the recombinant families are sufficient to place the QTL in the interval DXMit226-DXMit68, the

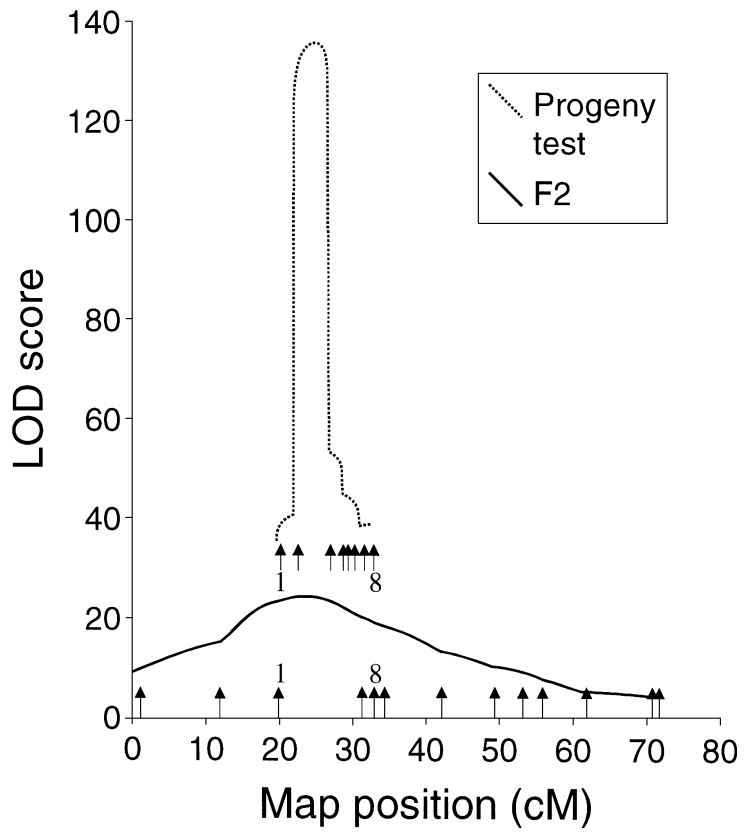

Fig. 2. LOD score plots obtained by interval mapping for the $\mathrm{X}$ chromosome using the progeny test data set or the F2 data set (Rance et al., 1997a). Markers labelled 1 and 8 are DXMit50 and DXMit25, respectively. Other markers used in the progeny test or the $\mathrm{F} 2$ are listed in the legend to Fig. 3 and Rance et al. (1997a), respectively.

recombinant family data would have been insufficient on their own to resolve the QTL further, had additional markers in this interval been available. Based on a drop in LOD score of 1 from the ML, a confidence interval for the QTL location is about $2 \mathrm{cM}$, and excludes the locations of the flanking markers. Estimates of the QTL effect obtained by ML or by analysis of variance (ANOVA) of the nonrecombinant individuals in the initial recombinant families are shown in Table 2. The estimated effects obtained from the two analyses agree closely. In proportional terms the estimated QTL effects in males and females are similar to our previous estimates (Rance et al., $1997 a, b$ ), i.e. the hemizygous male effect is about $18 \%$ of the low line population mean of $16.7 \mathrm{~g}$, and the heterozygous female effect is about $10 \%$ of the low line mean of $13.6 \mathrm{~g}$.

Table 1. Recombinants and markers used in the progeny test

\begin{tabular}{lcllr}
\hline \hline Interval & $\begin{array}{l}\text { No. of } \\
\text { recombinants }\end{array}$ & $\begin{array}{l}\text { Map } \\
\text { distance (cM) }\end{array}$ & $\begin{array}{l}\text { Mean no. of progeny test } \\
\text { litters per recombinant }\end{array}$ & $\begin{array}{l}\text { Total } \\
\text { progeny }\end{array}$ \\
\hline DXMit50-DXMit226 & 6 & $2 \cdot 3$ & $5 \cdot 5$ & 131 \\
DXMit226-DXMit68 & $18^{a}$ & $4 \cdot 8$ & $14 \cdot 8$ & 1175 \\
DXMit68-DXMit140/141/73 & 5 & $1 \cdot 9$ & $3 \cdot 0$ & 64 \\
DXMit140/141/73-DXMit144 & 6 & $2 \cdot 3$ & $1 \cdot 8$ & 27 \\
DXMit144-DXMit25 & 4 & $1 \cdot 5$ & $1 \cdot 5$ & 18 \\
\hline \hline
\end{tabular}

a One of these failed to reproduce.

${ }^{b}$ No recombinants were found between DXMit140, DXMit141 and DXMit73. 


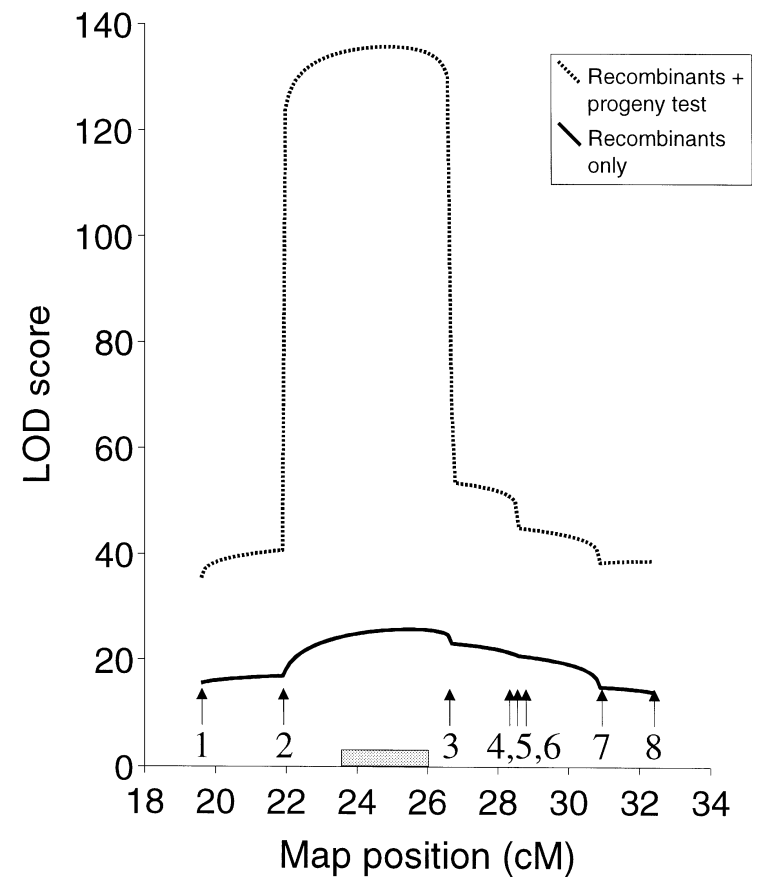

Fig. 3. LOD score plots obtained by interval mapping using the complete progeny test data including the families with the initial recombinants or the initial recombinants only. The marker labels are: 1, DXMit50; 2, DXMit226; 3, DXMit68; 4, DXMit140; 5, DXMit141; 6, DXMit73; 7, DXMit144; 8, DXMit25. The 95\% confidence intervals for the QTL position is indicated by the grey rectangle.

Table 2. Estimated heterozygous and hemizygous QTL effects

\begin{tabular}{lll}
\hline \hline & \multicolumn{2}{l}{ QTL effect $(\mathrm{g}) \pm \mathrm{SE}$} \\
\cline { 2 - 3 } Dataset (analysis method) & Females & Males \\
\hline Recombinants only (ANOVA) & $1 \cdot 3 \pm 0 \cdot 35$ & $3 \cdot 1 \pm 0 \cdot 33$ \\
Recombinants + progeny test (ML) & $1 \cdot 3 \pm 0 \cdot 15$ & $2 \cdot 8 \pm 0 \cdot 16$ \\
\hline \hline
\end{tabular}

The fitting of a two-QTL model led to an increase in LOD score over the single-QTL model of 1.7. This increase is significant at the nominal $5 \%$ level $\left(\chi^{2} 2 \mathrm{df}\right.$ $=7.8 ; P<0.05)$, but non-significant if corrected for multiple tests, assuming there are four tests corresponding to the four intervals in which a second QTL could occur (Fig. 3). The estimated effect of the major QTL is changed only slightly by fitting a second QTL, for which the estimated effect is about $-0.3 \mathrm{~g}$ in both males and females.

(ii) Integration of markers flanking the QTL into the EUCIB backcross

To facilitate the development of further molecular markers, the nearest microsatellite markers flanking the QTL (DXMit226 and DXMit68) were typed using mice from the European Collaborative Interspecific
Mouse Backcross (EUCIB), a resource for finemapping variants that segregate between Mus spretus and Mus domesticus. A panel of DNAs of 63 mice was chosen from the EUCIB cross that had been scored as recombinant between the microsatellite markers DXMit54 and DXMit87; these markers flank DXMit226 and DXMit68, respectively. The haplotypes of the panel placed DXMit226 between the STS DXMgc91 and the microsatellite DXMit82, while $D X M i t 68$ was placed between the STS $D X M g c 96$ and the Hprt gene (Fig. 1). However, the exact location of DXMit68 was somewhat ambiguous, possibly due to residual variation in the cross used to derive the EUCIB panel (Rhodes et al., 1998), and it may be distal to Hprt.

\section{(iii) Attempts at refining the map location}

A further 30 microsatellites in the region of DXMit226 and DXMit68 were tested for polymorphism. Of these, 5 polymorphic markers were found, but all were outside the target region according to the results of genotyping our recombinant panel. Based on mouse YAC STS-content data (Nusbaum et al., 1999) and information in public databases (MGChttp://www.hgmp.mrc.ac.uk/mbxbin/viewsam.pl; NCBI - http://www.ncbi.nlm.nih.gov/dbSTS; MGD http://mgd.hgmp.mrc.ac.uk/reports/mitmap), a set of STS was identified that mapped in or close to the target region. The sequences of the alleles in the high and low congenic lines along with introns 4, 5, 6 and 8 and other non-coding DNA of the Hprt gene and non-coding DNA of three other genes were determined (Table 3). Alignment of the sequences generated from forward and reverse primers revealed no differences in approximately $17000 \mathrm{bp}$.

\section{Discussion}

Progeny testing of recombinants from the initial screen led to greatly increased resolution of the QTL map position, and large changes in LOD score at the positions of markers. Some intervals can therefore be excluded as candidates for harbouring a major QTL. The only interval that can plausibly contain the major X-linked QTL is flanked by DXMit226 and DXMit68; there is little statistical support for an additional QTL in an adjacent region. These results confirm our previous findings that a single QTL explains a very high proportion $(\sim 20 \%)$ of the variance and selection response for body weight in the selection lines (Rance et al., 1997a,b), although it is not possible at this point to distinguish between one or more loci in the interval. The nearest markers flanking the QTL are approximately $5 \mathrm{cM}$ apart, a distance that is currently too large to allow the analysis of anonymous genes with the hope of finding potential candidates. However, the changes in LOD score between DXMit226 
Table 3. DNA segments sequenced in the region of the $Q T L$

\begin{tabular}{|c|c|c|}
\hline Locus name & $\begin{array}{l}\text { Length of } \\
\text { sequence (bp) }\end{array}$ & $\begin{array}{l}\text { Accession } \\
\text { no. }\end{array}$ \\
\hline \multicolumn{3}{|l|}{ STS (from MGC database) } \\
\hline$D X M g c 6$ & 140 & AF311623 \\
\hline$D X M g c 47$ & 149 & AF311624 \\
\hline$D X M g 83$ & 233 & AF311625 \\
\hline$D X M g c 92$ & 173 & AF311626 \\
\hline$D X M g c 93$ & 105 & AF311627 \\
\hline$D X M g c 94$ & 336 & AF311628 \\
\hline$D X M g c 95$ & 209 & AF311629 \\
\hline \multicolumn{3}{|l|}{ STSs (from MGD database) } \\
\hline DXJti2 & 457 & AF311612 \\
\hline DXJti3 & 287 & AF311611 \\
\hline$X 83577$ & 163 & AF311610 \\
\hline$C p-8-25-95-03$ & 168 & AF311608 \\
\hline MHAa97h7 & 159 & AF311609 \\
\hline 30.MМHAP16FRB12 & 184 & AF311614 \\
\hline 40.MМHAP76FLF4 & 177 & AF311613 \\
\hline 44.MМHAP34FRG11 & 197 & AF311607 \\
\hline \multicolumn{3}{|l|}{ Genes: } \\
\hline Api3 & 281 & AF311606 \\
\hline Gria3 & 291 & AF311605 \\
\hline Ant2 & 304 & AF311622 \\
\hline Hprt $5^{\prime}$ untranslated region & 411 & AF311615 \\
\hline Hprt $3^{\prime}$ untranslated region & 564 & AF311616 \\
\hline Hprt intron 4 & 3632 & AF311617 \\
\hline Hprt intron 5 & 4078 & AF311618 \\
\hline Hprt intron 6 & 3782 & AF311619 \\
\hline Hprt intron 7 & 247 & AF311620 \\
\hline Hprt intron 8 & 682 & AF311621 \\
\hline
\end{tabular}

and DXMit68 are so high that a dense map with complete resolution of recombinants should give LOD score changes at markers averaging about 5 . There are 18 recombinants between DXMit226 and DXMit68, so in future it may be possible to obtain a resolution of about $0.25 \mathrm{cM}$ between flanking markers. Assuming 80000 genes in the mouse genome (Antequera \& Bird, 1993), and that gene density on the X chromosome is $50 \%$ of that on the autosomes (Schuler et al., 1996), a $0.25 \mathrm{cM}$ region of the $\mathrm{X}$ chromosome is expected to contain about 7 genes. Such a number of genes could be analysed for sequence or expression differences.

There are currently no obvious candidate genes in the mapped region. Phenotypic studies of the effects of the QTL on several growth-related traits (Liu et al., 2001) suggest that the effects on growth are very general, and most easily detected on body weight (rather than, for example, body length). Specific organs affected to a greater or lesser extent by the QTL have not been seen. Although several other studies have found X-linked QTLs for growth-related traits (reviewed by Barsh et al., 2000), their phenotypes differ from our QTL, suggesting that different loci are involved.

As part of the effort to find new genetic markers in the region containing the QTL, sequences of sequence- tagged sites (STSs) and introns and other non-coding regions of several genes were determined for the high and low lines. However, in a total sequence length of over $17 \mathrm{~kb}$, involving the sequencing of both strands of DNA, no polymorphisms were found. As a check, the sequence of the PCR product from DXMit68 (a flanking marker that is polymorphic on the basis of gel electrophoresis) was determined, and differences in repeat lengths between the high and low line alleles were easily seen. The rate of nucleotide polymorphism in the interval containing the QTL is therefore extremely low in comparison with the average levels in humans and Drosophila (Li, 1997, chapter 9). In mice, the average level of single nucleotide polymorphism between pairs of inbred strains has been estimated to be close to 1 per $1000 \mathrm{bp}$ (Lindblad-Toh et al., 2000). Although there is some evidence of a reduced general level of variability on the $\mathrm{X}$ chromosome (reviewed by Hedrick \& Parker, 1997), our failure to detect any polymorphisms in about $17000 \mathrm{bp}$ seems to reflect a real lack of variation in the region rather than a sampling effect. This could be due to the specific region of the chromosome having a recent 'gene history', i.e. a recent coalescence time to the common ancestral sequence. If this is the case then the large Xlinked QTL segregating in an otherwise homozygous 
region suggests the possibility of a new mutation that arose relatively recently. New mutations of large effect are detected not infrequently in selection lines (Falconer \& Mackay, 1996), and their presence has been inferred in several instances where selection lines for body weight in mice have been analysed (Roberts, 1966; Bradford \& Famula, 1984; Keightley 1998). If the QTL is a recent mutation, it is unlikely to have occurred during the course of the selection experiment, since a large $\mathrm{X}$ chromosome effect was present in each of the three replicates of the experiment, which were later combined to form one replicate (Hastings \& Veerkamp, 1993). The lack of single nucleotide polymorphism has hampered progress in fine-scale mapping; current efforts are therefore aimed at finding new microsatellite markers in the region, and should be assisted by the availability of the complete mouse genome sequence.

We thank Andy Haynes and Claire Davison at the MRC UK Mouse Genome Centre for supplying EUCIB panel DNAs and for help with entering data into the $\mathrm{MBx}$ database, an anonymous referee for helpful comments on the manuscript, and the Medical Research Council for support.

\section{References}

Antequera, F. \& Bird, A. (1993). Number of CpG islands and genes in human and mouse. Proceedings of the National Academy of Sciences of the USA 90, 11995-11999.

Barsh, G. S., Farooqi, I. S. \& O'Rahilly, S. (2000). Genetics of body-weight regulation. Nature 404, 644-651.

Bradford, G. E. \& Famula, T. R. (1984). Evidence for a major gene for rapid postweaning growth in mice. Genetical Research 44, 293-308.

Breese, E. L. \& Mather, K. (1957). The organization of polygenic activity within a chromosome in Drosophila. I. Hair characters. Heredity 11, 373-395.

Darvasi, A. (1998). Experimental strategies for the genetic dissection of complex traits in animal models. Nature Genetics 18, 19-24.

Falconer, D. S. \& Mackay, T. F. C. (1996). Introduction to Quantitative Genetics, 4th edn. Harlow, Essex: Longman.

Frary, A., Nesbitt, T. C., Frary, A., Grandillo, S., van der Knaap, E., Cong, B., Liu, J. P., Meller, J., Elber, R., Alpert, K. B. \& Tanksley, S. D. (2000). fw2.2. a quantitative trait locus key to the evolution of tomato fruit size. Science 289, 85-88.

Haley, C. S. \& Knott, S. A. (1992). A simple regression method for mapping quantitative trait loci in line crosses using flanking markers. Heredity 69, 315-324.

Hastings, I. M. \& Veerkamp, R. F. (1993). The genetic basis of response in mouse lines divergently selected for body weight or fat content. I. The relative contributions of autosomal and sex-linked genes. Genetical Research 62, 169-175.

Hedrick, P. W. \& Parker, J. D. (1997). Evolutionary genetics and genetic variation of haplodiploids and X-linked genes. Annual Review of Ecology and Systematics 28, 55-83.
Kao, C. H., Zeng, Z. B. \& Teasdale, R. D. (1999). Multiple interval mapping for quantitative trait loci. Genetics 152, 1203-1216.

Keightley, P. D. (1998). Genetic basis of response to 50 generations of selection on body weight in inbred mice. Genetics 148, 1931-1939.

Keightley, P. D., Morris, K. H., Ishikawa, A., Falconer, V. M. \& Oliver, F. (1998). Test of candidate genequantitative trait locus association applied to fatness in mice. Heredity 81, 630-637.

Knott, S. A. \& Haley, C. S. (1992). Maximum likelihood mapping of quantitative trait loci using full sib families. Genetics 132, 1211-1222.

Li, W.-H. (1997). Molecular Evolution. Sunderland, MA: Sinauer.

Lindblad-Toh, K., Winchester, E., Daly, M. J., Wang, D. G., Hirschhorn, J. N., Laviolette, J. P., Ardlie, K., Reich, D. E., Robinson, E., Sklar, P., Shah, N., Thomas, D., Fan, J. B., Gingeras, T., Warrington, J., Patil, N., Hudson, T. J. \& Lander, E. (2000). Large-scale discovery and genotyping of single-nucleotide polymorphisms in the mouse. Nature Genetics 24, 381-386.

Liu, X., Bünger, L. \& Keightley, P. D. (2001). Characterisation of a major X-linked QTL influencing body weights in mice. Journal of Heredity in press.

Nelder, J. A. \& Mead, R. (1965). A simplex method for function minimization. Computer Journal 7, 308-313.

Nusbaum, C., et al. (1999). A YAC-based physical map of the mouse genome. Nature Genetics 22, 388-393.

Rance, K. A., Hill, W. G. \& Keightley, P. D. (1997a). Mapping quantitative trait loci for body weight on the $\mathrm{X}$ chromosome in mice. I. Analysis of a reciprocal F2 population. Genetical Research 70, 117-124.

Rance, K. A., Heath, S. C. \& Keightley, P. D. (1997b). Mapping quantitative trait loci for body weight on the $\mathrm{X}$ chromosome in mice. II. Analysis of congenic backcrosses. Genetical Research 70, 125-133.

Rhodes, M., Straw, R., Fernando, S., Evans, A., Lacey, T., Dearlove, A., Greystrong, J., Walker, J., Watson, P., Weston, P., Kelly, M., Taylor, D., Gibson, K., Mundy, C., Bourgade, F., Poirier, C., Simon, D., Brunialti, A. L. B., Montagutelli, X., Guenet, J.-L., Haynes, A. \& Brown, S. D. M. (1998). A high-resolution microsatellite map of the mouse genome. Genome Research 8, 531-542.

Roberts, R. C. (1966). The limits to artificial selection for body weight in the mouse. II. The genetic nature of the limits. Genetical Research 8, 361-375.

Routman, E. \& Cheverud, J. (1994). A rapid method of scoring simple sequence repeat polymorphisms with agarose gel electrophoresis. Mammalian Genome 5, 187-188.

Schuler, G. D., et al. (1996). A gene map of the human genome. Science 274, 540-546.

Sharp, G. L., Hill, W. G. \& Robertson, A. (1984). Effects of selection on growth, body composition and food intake in mice. I. Responses in selected traits. Genetical Research 43, 75-92.

Shrimpton, A. E. \& Robertson, A. (1988). The isolation of polygenic factors controlling bristle score in Drosophila melanogaster. I. Allocation of third chromosome sternopleural bristle effects to chromosome sections. Genetics 118, 437-443.

Thoday, J. M. (1961). Location of polygenes. Nature 191, 368-370.

Weber, K., Eisman, R., Morey, L., Patty, A., Sparks, J., Tausek, M. \& Zeng, Z. B. (1999). An analysis of polygenes affecting wing shape on chromosome 3 in Drosophila melanogaster. Genetics 153, 773-786. 